\title{
Validación de modelos hidrodinámicos de tres modelos topológicos de lagunas facultativas secundarias
}

\author{
Validation Hydrodynamic Models of Three Topological Models of Secondary \\ Facultative Ponds
}

\author{
Aponte-Reyes Alxander \\ Universidad del Valle \\ Grupo de Investigación Saneamiento Ambiental, Colombia \\ Correo:alexander.aponte.reyes@correounivalle.edu.co
}

Información del artículo: recibido: febrero de 2013, reevaluado: agosto de 2013, aceptado: febrero de 2014

\section{Resumen}

Se desarrolló una metodología para analizar las condiciones de frontera, el tamaño de la malla y el modelo de turbulencia más ajustado de un modelo matemático de la mecánica computacional de fluidos, (CFD, computational fluid mechanics) que explicara el comportamiento hidrodinámico de topologías de lagunas facultativas secundarias, LFS, construidas a escala piloto: laguna convencional, LC, laguna bafles, LB y laguna bafles-mallas, LBM. Para la validación se realizaron estudios de dispersión en campo, tomando muestras al interior y a la salida de las unidades piloto, la información se utilizó para llevar a cabo simulaciones de los modelos CFD de las tres topologías. Los tamaños de malla evaluados variaron entre 500,000 y 2,000,000 de elementos. La condición de frontera en superficie Pared mostró un buen comportamiento cualitativo y el modelo de turbulencia $\kappa-\varepsilon$ Low Reynolds arrojó buenos resultados. La biomasa contenida en LFS genera interferencias sobre los estudios de dispersión y debe considerarse en la evaluación del modelado CFD, los tiempos de inyección del trazador, su concentración a la entra$\mathrm{da}$, el efecto del viento sobre los modelos CFD y los caudales adoptados como base para el modelado son parámetros que deben tenerse en cuenta para la validación y calibración de los modelos CFD.

\section{Descriptores:}

- dinámica computacional de fluidos

- lagunas facultativas secundarias

- estudios de dispersión

- hidrodinámica 


\begin{abstract}
A methodology was developed to analyze boundary conditions, the size of the mesh and the turbulence of a mathematical model of CFD, which could explain hydrodynamic behavior on facultative stabilization ponds, FSP, built to pilot scale: conventional pond, $C P$, baffled pond, BP, and baffled-mesh pond, BMP. Models dispersion studies were performed in field for validation, taking samples into and out of the FSP, the information was used to carry out CFD model simulations of the three topologies. Evaluated mesh sizes ranged from 500,000 to 2,000,000 elements. The boundary condition in Pared surface-free slip showed good qualitative behavior and the turbulence model $\kappa-\varepsilon$ Low Reynolds yielded good results. The biomass contained in LFS generates interference on dispersion studies and should be taken into account in assessing the CFD modeling, the tracer injection times, its concentration at the entrance, the effect of wind on CFD, and the flow models adopted as a basis for modeling are parameters to be taken into account for the CFD model validation and calibration.
\end{abstract}

\section{Introducción}

El comportamiento de un fluido dentro de un sistema de tratamiento de aguas residuales, STAR, como las lagunas facultativas secundarias, LFS, es objeto de múltiples estudios (Alvarado et al., 2012; Sah, 2009; Shilton et al., 2008) que buscan mejorar su funcionamiento (Mara, 2004); se han realizado esfuerzos especiales para la comprensión de su hidrodinámica a través del uso de herramientas computacionales y trabajo de campo en estudios de dispersión. El estudio de las fuerzas que actúan sobre el agua (Ji, 2008) puede abordarse mediante la dinámica computacional de fluidos (CFD, computational fluid mechanics), que utiliza algoritmos numéricos para predecir fenómenos como el comportamiento de un fluido, la transferencia de calor, la transferencia de masa, cambios de fase, reacciones químicas, entre otros, resolviendo las ecuaciones matemáticas que gobiernan estos procesos (ANSYS, 2006) y suponiendo que un sistema determinado se rige por las leyes de conservación de estas características (Banda, 2007; Bird et al., 2006; Patankar, 1980). En CFD las fronteras son los límites de los modelos físicos y a ellas se les asignan valores iniciales para resolver las ecuaciones diferenciales que los determinan. La definición acertada de las fronteras y sus condiciones es importante pues se supone que lo que sucede al interior del modelo estudiado depende de ellas (Jakeman et al., 2006). Para la definición del método de solución numérica los elementos que se tienen en cuenta en la CFD son: el modelo matemático, el método de discretización, el sistema de coordenadas y vectores, la malla (que define el dominio, 2D o 3D), las aproximaciones finitas, el método de solución y los criterios de convergencia (Ferziger y Peric, 2002). Para las fronteras se definen las condiciones asociadas a masa, momento y energía de las ecuaciones diferenciales correspondientes. Cada software o modelo matemático aborda las fronteras en forma particular, de allí que su selección deba ser acertada para obtener los resultados más próximos a la solución del problema a analizar. La siguiente ecuación resume el balance de cantidad de movimiento aplicado en CFD.

$$
\begin{array}{ccc}
\frac{\partial}{\partial t}(\rho \phi) & +\frac{\partial}{\partial x_{j}}\left(\rho u_{j} \phi\right) & \frac{\partial}{\partial x_{j}}\left(\Gamma \frac{\partial \phi}{\partial x_{j}}\right) \\
\text { término de } & \text { término } \\
\text { acumulación } & \text { convectivo } & \text { difusivo }
\end{array} \quad \begin{gathered}
\text { término } \\
\text { fuente }
\end{gathered}
$$

donde $\rho$ es la densidad $\left(\mathrm{ML}^{-3}\right), x$ es una variable que puede ser vectorial o escalar, $u\left(\mathrm{Lt}^{-1}\right)$ es la velocidad en la dirección $j, \Gamma\left(\mathrm{M}^{-2} \mathrm{t}^{-1}\right)$ es el coeficiente de difusividad $\mathrm{y}$ $S\left(\mathrm{ML}^{-3} \mathrm{t}^{-2}\right)$ es un término fuente para la variable $\phi$. Para un fluido incompresible bajo una condición de estado no estacionario, la ecuación de continuidad viene definida por:

$\frac{\partial \rho}{\partial t}+\frac{\partial(\rho u)}{\partial x}+\frac{\partial(\rho v)}{\partial y}+\frac{\partial(\rho w)}{\partial z}=0$

donde $u, v, w$ corresponden a las velocidades $\left(\mathrm{Lt}^{-1}\right)$ en las direcciones $x$, y y $z$ y $\rho\left(\mathrm{ML}^{-3}\right)$ a la densidad del fluido. En fluidos incompresibles newtonianos los esfuerzos viscosos son proporcionales a las tasas de deformación (Branncock, 2003). El movimiento de remolino contribuye apreciablemente al proceso de transferencia de masa, este fenómeno genera difusividades 
de remolino de cantidad de movimiento, de calor y masa que pueden ser calculados (Treybal, 1980). Los flujos turbulentos pueden analizarse como un flujo laminar no turbulento en el que se suceden fluctuaciones rápidas y aleatorias alrededor del valor promedio. Los modelos de flujo turbulento tienen la tarea de explicar esas fluctuaciones y lo hacen sumando o adicionando coeficientes. La turbulencia incluye la energía cinética y la energía cinemática (Patankar, 1980). Reynolds planteó que en flujos turbulentos los promedios de los esfuerzos son iguales a 0 . De este planteamiento se sigue que la turbulencia se relaciona con los esfuerzos de Reynolds a través de la siguiente expresión $R_{i j}=-\rho u_{i} u_{j}$ Para la explicación del movimiento del fluido se utilizan las ecuaciones de Navier-Stokes promediadas en Reynolds, (RANS, Reynolds-averaged Navier-Stokes equations), ecuación 3, las cuales obligan a modelar los esfuerzos de Reynolds usando CFD.

$$
\frac{\partial\left(\rho U_{i}\right)}{\partial t}+\frac{\partial}{\partial x_{j}}\left(\rho U_{i} U_{j}\right)=-\frac{\partial p}{\partial x_{i}}+\frac{\partial}{\partial x_{j}}\left(\mu \frac{\partial U_{i}}{\partial x_{j}}-\rho \overline{u_{i} u_{j}}\right)+S_{M}
$$

El modelado de la turbulencia de los esfuerzos de Reynolds relaciona los componentes de los esfuerzos de Reynolds desconocidos con propiedades del flujo para que exista un número de ecuaciones que permitan calcularla. Usualmente estas cantidades son la energía cinética turbulenta, $\kappa,\left(\mathrm{L}^{2} \mathrm{t}^{-2}\right)$ y la energía de disipación turbulenta, $\varepsilon,\left(\mathrm{L}^{2} \mathrm{t}^{-3}\right)$. Existe software de CFD que resuelve las ecuaciones correspondientes con base en el modelo de turbulencia adoptado. Uno de los fenómenos presentes en sistemas de tratamiento natural, STN, es el ingreso de flujos a los cuerpos de agua. Dichos flujos se transportan y dispersan a través de los vórtices más grandes hasta los más pequeños donde la difusión molecular puede actuar y la habilidad de los flujos turbulentos con altos números de Reynolds permite que la mezcla molecular exista. La viscosidad turbulenta se puede entender en tres momentos: entrada, dispersión (mezcla) y difusión; debe considerarse que esa difusión opera en la medida que primero actúa la viscosidad y luego la dispersión. La mezcla puede ser pasiva y podría considerarse independiente de la dinámica del fluido (Dimotakis, 2005), sin embargo la intensidad de remolino varía con la distancia (Bird et al., 2006). La existencia de software potentes permite evaluar modelos de turbulencia que dan resultados dependiendo del tipo de reactor que se esté analizando (Han et al., 2012). Por lo anterior, la viscosidad turbulenta se convierte en uno de los parámetros más importantes en el estudio de la hidrodinámica empleando CFD. Cada uno de los modelos tiene sus pros y sus contras cuando de predecir la dinámica de un fluido se trata.

El cambio de masa de una sustancia en un fluido con respecto al tiempo, sumado a las derivadas parciales del flujo en las direcciones $x, y$ y $z$ en sus direcciones correspondientes (por convección y por difusión) es igual a la tasa de generación o destrucción de la masa de la especie química si esta es reactiva. La derivada de la masa de la sustancia en las direcciones $x, y$ y $z$ multiplicada por el coeficiente de difusión es igual a la difusión de la sustancia. La convección corresponde al flujo de masa generado por un campo de velocidad; mientras que la difusión es el flujo generado por gradientes de concentración (masa) de la sustancia/ especie y puede expresarse por la ley de difusión de Fick que determina un coeficiente de difusión que afecta la especie en las direcciones $x, y$ y $z$ (Patankar, 1980). A través de la CFD se pueden simular sustancias o especies como una propiedad o un fenómeno como el transporte y su transformación, de allí que pueda incorporar al componente hidrodinámico fenómenos bioquímicos o de reacción que involucran sustratos y biomasa. Para el caso del transporte, la ecuación para una sustancia $\phi$ se convierte en:

$\frac{\partial(\rho \phi)}{\partial t}+\nabla \cdot(\rho U \phi)=\frac{\partial}{\partial x_{j}}\left(\rho\left(\mu+\frac{\mu_{t}}{S c_{t}}\right) \frac{\partial \phi}{\partial x_{j}}-\rho \overline{u_{j} \varphi}\right)+S_{\phi}$

La simulación mediante CFD debe ser validada (Laín, 2007) y para esto existen estrategias que incluyen el seguimiento de elementos flotantes y los estudios de trazadores; adicionalmente, la construcción de sistemas a escala piloto son de gran ayuda para el ejercicio de validación de los modelos CFD (Shilton, 2001; Shilton et al., 2008). Una LFS basa su rendimiento en el flujo de sustratos, los mecanismos de transporte de ese sustrato a lo largo del reactor y el contacto entre el sustrato y la biomasa activa (microorganismos), además de los factores atmosféricos (viento y radiación solar). Para representar reactores de flujo continuo existen modelos correspondientes a patrones de flujo ideales y no ideales asociados a un reactor. En el caso de los flujos ideales se reconocen el modelo de flujo pistón, FP, y el modelo de flujo completamente mezclado, FCM, mientras que entre los flujos no ideales se considera el flujo pistón con dispersión axial, FD, o el de tanques mezclados en serie, TIS, como los más representativos (Crites y Tchobanoglous, 2000). El tiempo de retención hidráulica se considera el lapso de tiempo entre la entrada de una partícula de agua a un reactor y la salida del mismo. El tiempo de retención nominal se estima con base 
en la ecuación 5 , esta considera que todas las partículas tienen el mismo tiempo de retención y representa un valor ideal, no considera las fluctuaciones mencionadas previamente.

$t_{n}=\frac{V}{Q}$

Estas fluctuaciones hacen que existan lotes de partículas con tiempos de retención distintos, los cuales pueden graficarse y constituyen la distribución de estos tiempos de residencia del fluido, que se conoce como las distribuciones de la edad a la salida $\mathbf{E}$ y $\mathbf{F}$, comúnmente se grafican y se conocen como curvas DTR (distribución de tiempos de residencia). Para la determinación de las DTR se realizan estudios de dispersión que consisten en el suministro al reactor de una sustancia no reactiva al fluido, y posteriormente se espera la respuesta a dicho estímulo en la salida del reactor (Yánez, 1993); al graficar las concentraciones medidas vs el tiempo transcurrido desde el suministro del trazador se construye la denominada curva $\mathrm{C}$ (figura 1).

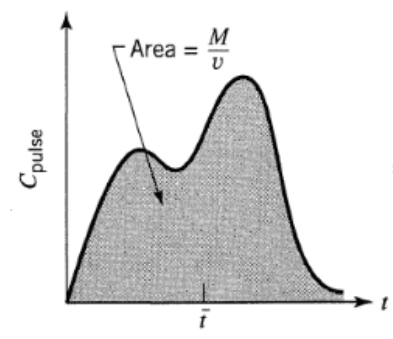

Tomado de LEVESPIEL, 2004. p. 263

$$
\left(\begin{array}{l}
\text { Área bajo la curva } \\
\text { C impulso }
\end{array}\right) ; \quad \mathrm{A}=\int_{0}^{\infty} C d t \cong \sum_{i} C_{i} \Delta t_{i}=\frac{M}{v}
$$

Figura 1. Curva C. C vs t

Fuente: Levenspiel (1999)

El área bajo la curva corresponde a la concentración del trazador en el reactor en un tiempo $t$.

$$
A=\int_{0}^{\infty} C d t \cong \sum_{i} C_{i} \Delta t_{i}=\frac{M}{v}
$$

donde:

$C=$ concentración del trazador en el tiempo, $\mathrm{ML}^{-3}$

$M=$ masa del trazador inyectada, $M$

$v=$ flujo volumétrico del trazador a la entrada, $\mathrm{L}^{3} \mathrm{t}^{-1}$
La curva $E$ se crea multiplicando cada valor de la curva C por $v / M$ (el área bajo la curva) y graficando contra el tiempo.

$$
E=\frac{C_{\text {pulse }}}{M / v}
$$

La curva también recibe el nombre de curva de densidad, pues acumula los tiempos de retención de las partículas en el reactor. Para comparar la curva $E$ obtenida del comportamiento del reactor real, con las curvas $E$ de comportamiento de patrones de flujo ideal, se debe normalizar con respecto al tiempo (figura 2).

$E_{\theta}=\bar{t} E=\frac{V}{v} \frac{C_{\text {pulse }}}{M / v}=\frac{V}{M} C_{\text {pulse }}$

donde $\bar{t}$ es el tiempo de retención hidráulico y se obtiene:

$\bar{t}=\frac{\int_{0}^{\infty} t C d t}{\int_{0}^{\infty} C d t} \cong \frac{\sum t_{i} C_{i} \Delta t_{i}}{\sum C_{i} \Delta t_{i}}=\frac{V}{v}$

La $E$ normalizada se grafica con respecto al tiempo normalizado, $\theta$.

$\theta=t_{i} / \bar{t}$

Dependiendo del flujo dentro del reactor la curva $E$ normalizada adoptará las siguientes formas:

\section{Desarrollo}

La investigación se realizó en la Estación de investigación de aguas residuales y reuso de Ginebra, EIG, localizada en el municipio de su mismo nombre, en el Valle del Cauca, Colombia, a $3^{\circ} 43^{\prime} 50^{\prime \prime}$ latitud norte y $76^{\circ} 16^{\prime} 20^{\prime \prime}$ longitud este, a 1040 m.s.n.m. La temperatura promedio es de $23^{\circ} \mathrm{C}$ y la precipitación anual promedio de $1280 \mathrm{~mm}$. Se hizo seguimiento a tres LFS con distintas configuraciones físicas, vea la tabla 1. El diseño de las unidades piloto se realizó empleando la metodología de carga orgánica superficial (Mara, 2004) aplicando un análisis de incertidumbre (Von-Sperling, 1996) con base en la propuesta de Banda (2003). Se empleó la técnica de estímulo-respuesta empleando un pulso de rodamina water tracing, RWT, como trazador para los tres pilotos en estudio, LBM, LC y LB. La con- 
centración de la RWT se determinó midiendo su fluorescencia utilizando un fluorómetro Turner M 8000-010. Los niveles mínimo y máximo de detección de RWT en el equipo son $0.4 \mathrm{ppb}$ y $300 \mathrm{ppb}$, respectivamente.

Las LFS fueron alimentadas con efluentes de la laguna anaerobia que trata el agua residual de la cabecera municipal de Ginebra; se presentó arrastre de lodos de la laguna anaerobia y los caudales a la entrada presentaron fluctuaciones. La figura 4 ilustra los pilotos y los puntos de muestreo. En P1 y P2 se tomaron muestras a dos profundidades, $0.05 \mathrm{~m}$ y $0.75 \mathrm{~m}$; fueron muestras puntuales en recipientes plásticos opacos con capaci
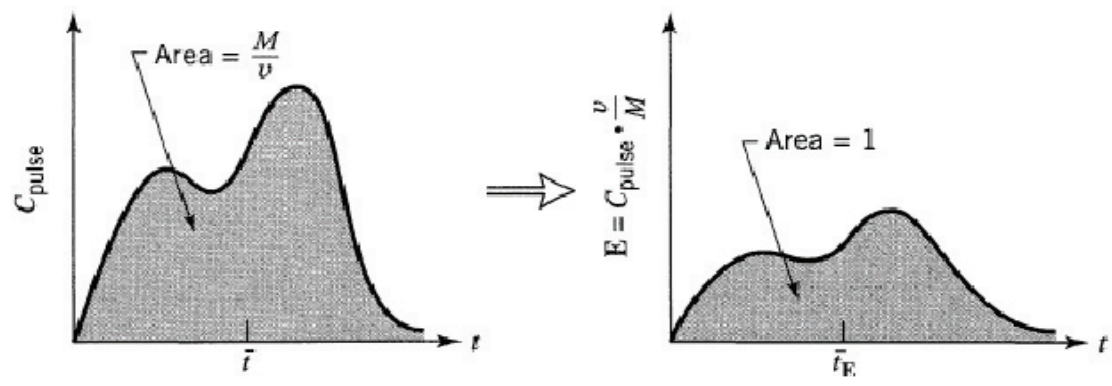

Tomado de LEVESPIEL, 2004. p. 263
Figura 2. Curva E. Evs t Fuente: Levenspiel (1999)
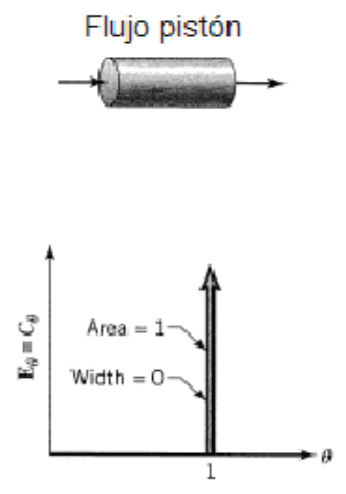

Mezcla completa
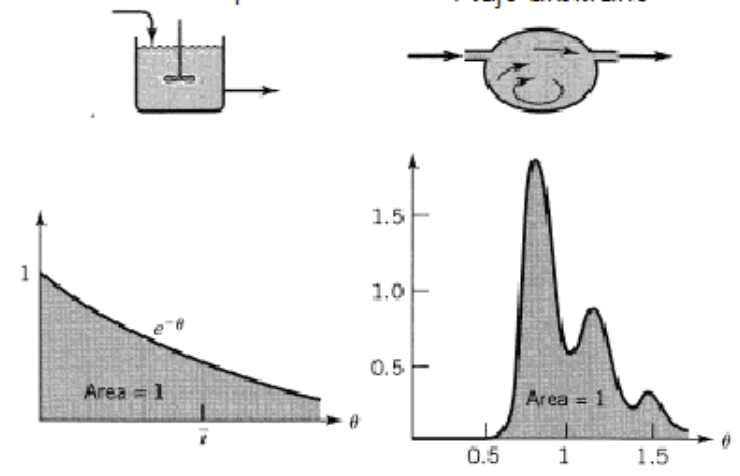

Tomado de LEVESPIEL, 2004. p. 266

Figura 3. Curva $\mathrm{E}_{\theta}$ vs $\theta$ Fuente: Levenspiel (1999)

Tabla 1. Características de las unidades piloto

\begin{tabular}{lccc}
\hline \multicolumn{1}{c}{ Características de diseño } & LFS 1. Bafles Mallas. LBM & LFS 2. Convencional. LC & LFS 3. Bafles. LB \\
\hline \multicolumn{1}{c}{ Modificación } & $\begin{array}{c}\text { Construcción bafles } \\
\text { e instalación de mallas } \\
\text { a L/3 y 2/L3 }\end{array}$ & & $\begin{array}{c}\text { Construcción de dos } \\
\text { bafles a L/3 y 2/L3 }\end{array}$ \\
\hline Caudal (L/s) & 0.275 & 0.275 & 0.275 \\
Altura (m) & 1.39 & 1.31 & 1.32 \\
Ancho espejo de agua (m) & 5.81 & 5.64 & 5.58 \\
Largo espejo de agua $(\mathrm{m})^{*}$ & 17.46 & 17.49 & 17.57 \\
Volumen $\left(\mathrm{m}^{3}\right)^{*}$ & 99.01 & 92.01 & 94.64 \\
TRH (días) & 4.17 & 3.99 & 3.98 \\
Área $\left(\mathrm{m}^{2}\right)$ & 45.08 & 49.25 & 48.16 \\
Carga aplicada $\left(\mathrm{kg} \mathrm{DBO}^{*} \mathrm{Ha}^{-1}\right)$ & 271.23 & 278.92 & 280.64 \\
\hline
\end{tabular}

* Las diferencias responden a condiciones constructivas. Se admitieron diferencias menores que 5\% en las variables TRH, área y carga aplicada 


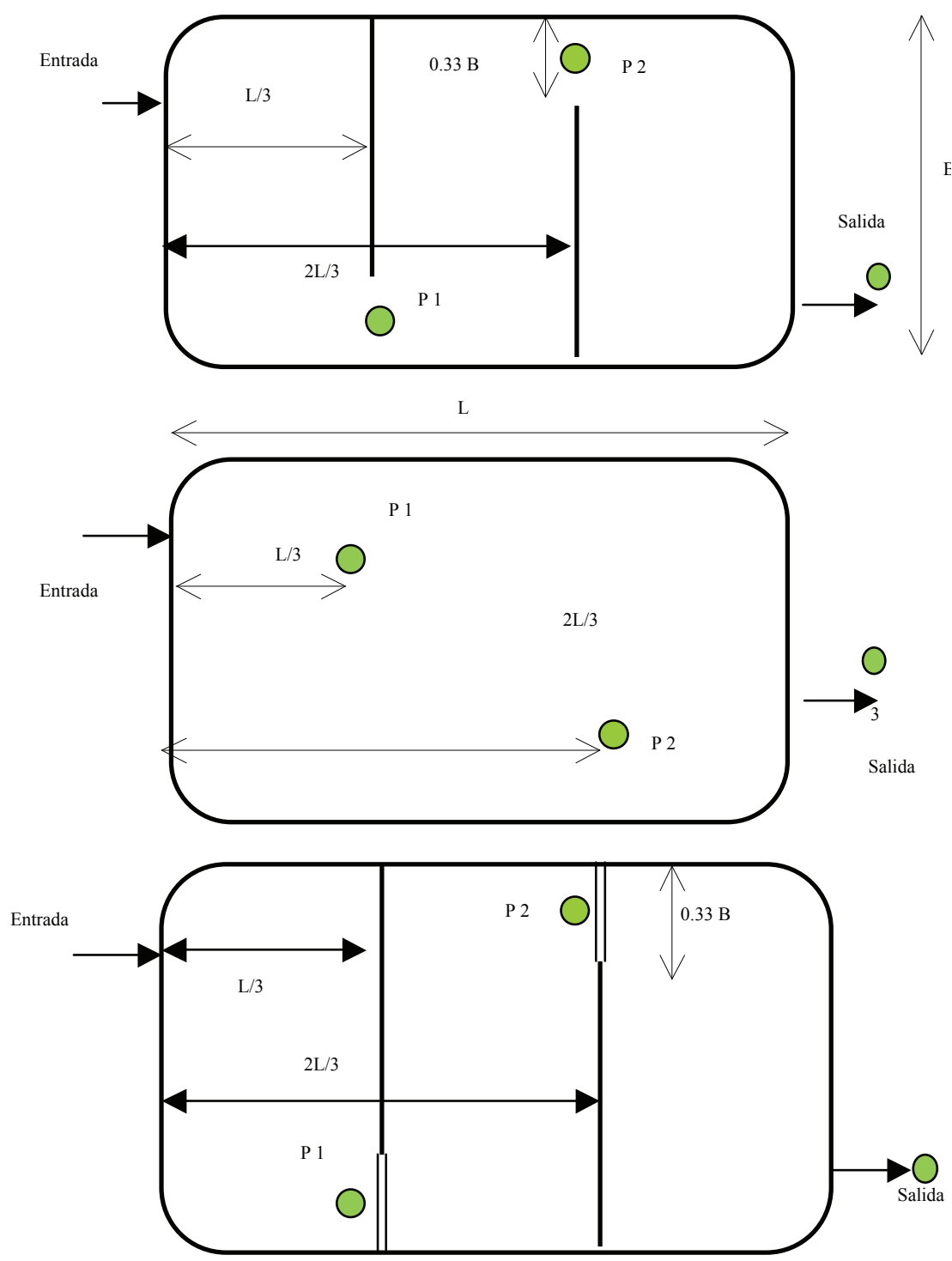

Figura 4. Esquema de unidades piloto y puntos de muestreo. De abajo a arriba, LBM, LC y LB

dad entre 80-100 mL. Las muestras se almacenaron a temperatura ambiente en un lugar oscuro durante máximo 4 horas hasta el momento de su análisis. La frecuencia de muestreo se presenta en la tabla 2, el rango de muestreo se propuso de acuerdo con las recomendaciones de Yánez (1993). La cantidad de trazador adicionado a los pilotos se determinó teniendo en cuenta lo recomendado por Kilpatrick (1970) y por Yánez (1993), con base en los picos de concentración esperados. El volumen de Rodamina aplicado fueron $19.21 \mathrm{~mL}$. La concentración de la solución de RWT inyectada a cada piloto fue de $760 \mathrm{mg}-\mathrm{L}^{-1}$. Se registró información de radiación solar, viento y precipitación mediante una esta- ción meteorológica portátil, EMP, Precision Weather Station DAVIS VANTAGE PRO2 PLUS, a una distancia de $1.5 \mathrm{~m}$ de los piloto y a una E altura de $4.0 \mathrm{~m}$; el anemómetro de la EMP se instaló a $5.0 \mathrm{~m}$ de altura. La estación tomó información durante todo el tiempo que duró la investigación y registró cada 30 minutos las variables de interés (tabla 3).

Aunque se realizó una serie de 3 estudios de dispersión, solo se presenta la información del segundo. La duración del estudio fue 3.5 veces el tiempo de retención nominal (Yánez, 1993). Los caudales de alimentación fueron medidos en forma volumétrica en las cajas de entrada a cada piloto y en la caja de salida de los mismos; las válvulas de ingreso de agua a cada laguna se calibraron cada hora durante el tiempo de duración del estudio de dispersión. Previo al estudio se hicieron mediciones del efluente de cada laguna para estimar la condición inicial de fluorescencia, background, y ajustar las lecturas de RWT (Kilpatrick y Wilson, 1989) medidas, restando este valor a cada medición. Los valores de background

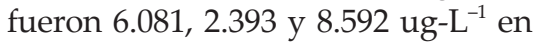
LBM, LC y LB, respectivamente. De acuerdo con la cantidad aplicada a la entrada y con la medición en puntos intermedios y a la salida de los pilotos se construyeron curvas de concentración vs tiempo.

Para el estudio de dispersión 2 todas las muestras a la salida de los pilotos se centrifugaron e hicieron ajustes a los valores de RWT medidos. El análisis de la información obtenida mediante el estudio de trazadores se analizó mediante la construcción de las curvas concentración vs t en cada punto de toma de muestra de los pilotos, así como la curva $E(\theta)$ vs $\theta$ (figura 5). Las tablas 4 y 5 resumen las variables hidráulicas que permiten analizar las curvas DTR, así como los modelos de dispersión y tanques en serie obtenidos para el ensayo 2. El modelado CFD se hizo empleando el software FLUENT $\AA$, usado en otras experiencias de simulación; las tablas 4 y 5 resumen el set up utilizado en la condición de frontera seleccionada y para simular la inyección del trazador. En los mode- 


\begin{tabular}{|c|c|c|c|c|c|}
\hline Fecha & $\begin{array}{c}\text { Día de } \\
\text { muestreo }\end{array}$ & Periodo & Núm. de horas & $\begin{array}{c}\text { Frecuencia } \\
\text { horaria }\end{array}$ & $\begin{array}{c}\text { Datos por } \\
\text { punto }\end{array}$ \\
\hline 20 Sep. 10:00 a 20 Sep. 22:00 & 1 & $0 \mathrm{~h}-12 \mathrm{~h}$ & 12 & 2 & 7 \\
\hline 21 Sep. 22:00 a 23 Sep. 14:00 & 1 a 2 & $12 \mathrm{~h}-52 \mathrm{~h}$ & 40 & 4 & 10 \\
\hline 23 Sep. 14:00 a 25 Sep. 2:00 & 2 a 4 & $52 \mathrm{~h}-88 \mathrm{~h}$ & 36 & 2 & 18 \\
\hline 25 Sep. 2:00 a 27 Sep. 6:00 & 4 a 6 & $88 \mathrm{~h}-140 \mathrm{~h}$ & 52 & 4 & 13 \\
\hline 27 Sep. 6:00 29 Sep. 10:00 & 6 a 8 & $140 \mathrm{~h}-156 \mathrm{~h}$ & 16 & 8 & 2 \\
\hline 29 Sep. 10:00 a 4 Oct. 10:00 & 8 a 14 & $156 h-348 h$ & 192 & 12 & 16 \\
\hline & & & 348 & & 66 \\
\hline & & & \multicolumn{2}{|c|}{ Número de puntos } & 5 \\
\hline & & & \multicolumn{2}{|c|}{ Número de lagunas } & 3 \\
\hline & & & \multicolumn{2}{|c|}{ Número total de datos } & 990 \\
\hline
\end{tabular}

\begin{tabular}{lcc}
\hline \multicolumn{1}{c}{ Variable } & Unidades & Observaciones \\
\hline $\begin{array}{l}\text { Temperatura interna (consola) } \\
\begin{array}{l}\text { Temperatura externa (Sensor } \\
\text { de la EMP) }\end{array}\end{array}$ & ${ }^{\circ} \mathrm{C}$ & \\
$\begin{array}{l}\text { Dirección del viento } \\
\text { Magnitud del viento }\end{array}$ & -- & $\begin{array}{l}16 \text { puntos cardinales, 22.5 } 5^{\circ} \text { entre } \\
\text { cada uno de ellos }\end{array}$ \\
Radiación solar & $\mathrm{m} / \mathrm{s}$ & \\
\hline
\end{tabular}

Tabla 2. Frecuencia de muestreo estudio de dispersión
Tabla 3. Variables meteorológicas medidas en la EIG

Fuente: DAVIS (2008)

los se definieron puntos de monitoreo de la concentración de RWT correspondientes a los de las muestras tomadas en los pilotos. Los modelos se construyeron empleando el software Gambit y el ICEM, se analizaron mallas gruesas ( $<300,000$ elementos), finas $(400,000$ a $1,000,000$ de elementos) y refinadas ( $>1,500,000$ elementos) y distintos tipos de frontera para la superficie. Para cada punto intermedio se analizaron los valores de concentración modelados y reales (muestras de los pilotos) comparando las curvas y los valores obtenidos; para esto se aplicó una prueba $t$-student pareada para hallar diferencias o similitudes entre los ensayos de trazadores; se empleó el software SPSS ${ }^{\circledR}$. La información obtenida con la EMP se relacionó con información del comportamiento de los pilotos en cuanto a su hidrodinámica. Proporcionó información de vientos en lo relacionado con las simulaciones realizadas en CFD. En el desarrollo del proceso de validación el modelo CFD fue corrido hasta el momento en el cual se alcanzó la concentración máxima de RWT, así como el valor de la misma.

\section{Análisis y discusión de resultados}

Los pilotos se comportaron de manera similar, observándose retraso en la salida del trazador en el caso de la
LBM; sin embargo por la forma de la curva se evidencian cortos circuitos a manera de pulsos que gradualmente se hacen menos pronunciados (figura 5). El comportamiento más irregular correspondió al de la LB; la LC también mostró un comportamiento con cortos circuitos, pero con una clara tendencia a RCM. Los dispositivos en LBM y LB modificaron la condición de flujo esperada en una LC, aunque los pronunciados picos en el inicio del ensayo muestran cortos circuitos de gran magnitud.

Las LFS tuvieron tendencia a RCM en concordancia con lo planteado por varios autores (Short et al., 2010; Polprasert y Battharai, 1985), los números de dispersión fueron similares para LBM y LB. La recuperación de trazador en LC superó la de LBM y LB, esto pudo tener como explicación una mayor velocidad de transporte del trazador, una condición de RCM más acentuada o una menor presencia de microalgas en este piloto, indicando un corto circuito mayor en LC que en las otras dos LFS. La dispersión en LC fue mayor que la hallada para LBM y LB, pero en los tres casos el flujo tendió a ser arbitrario. En cuanto al modelo de tanques mezclados en serie, la LBM alcanzó un valor de $n$ cercano a dos unidades, mientras que LB y LC tuvieron valores de 1.59 y 1.43 , respectivamente. No era de esperarse un comportamiento de LC similar al 
a)

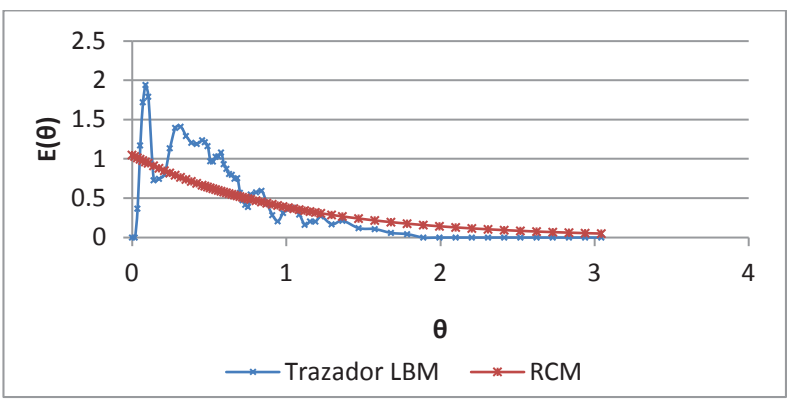

b)

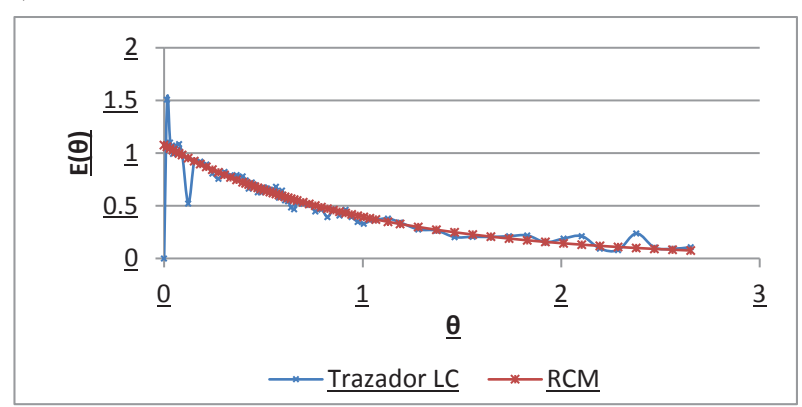

c)

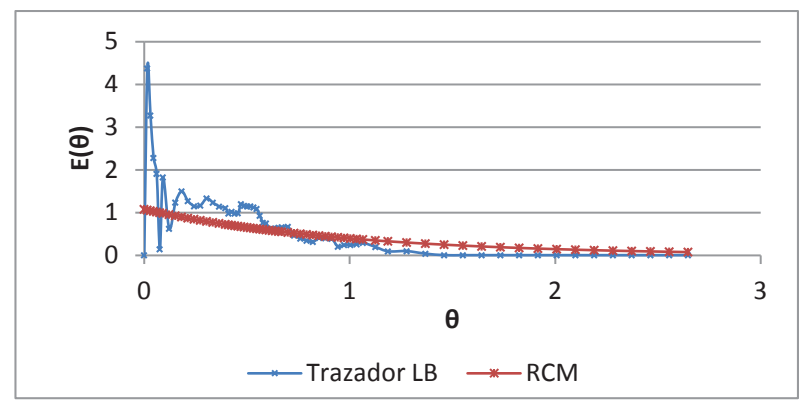

Figura 5. Curvas $\mathrm{E}\left({ }_{\theta}\right)$ vs $\theta$

de LB, sobre todo porque para el segundo los bafles generan una condición de compartimentalización del reactor. La LC fue la base para la estructuración del modelo hidrodinámico utilizado para las otras dos LFS. Como etapas previas a la selección de la malla se realizaron pruebas para explorar el tipo de frontera en la superficie. De las tres fronteras se seleccionaron dos tipos, velocidad de entrada, VE, y pared, $\mathrm{P}$, pues la frontera presión de salida, PS, no arrojó resultados satisfactorios desde el punto de vista cualitativo, figura 6, ni cuantitativo para los puntos de monitoreo. Finalmente, se decidió por Pared-Free Slip, que permitió simulaciones de viento sobre la superficie; además, ha sido empleada con éxito por otros investigadores (Banda, 2007). Los contornos de velocidad presentados en la figura 6 dan una idea de los recorridos preferencia- les del fluido para los tres tipos de frontera. En el caso de PS se observa un recorrido preferencial del fluido en los bordes de la frontera del modelo, comportamiento sensiblemente distinto al observado para VE y $\mathrm{P}$ en donde se observa un transporte que se dispersa en la superficie del plano mostrado en la figura, algo que fue posible observar directamente en los pilotos.

La malla seleccionada, tamaño de elemento característico $0.10 \mathrm{~m}$, correspondió a aquella en la que durante un modelado en estado estacionario tuvo puntos de monitoreo de velocidad (seguimiento del valor de velocidad en un punto de la laguna) con poca variación, sumado esto a la magnitud en los valores residuales de las soluciones numéricas para las variables continuidad y velocidad en $x, y$ y $z$ inferiores a $10^{-4}$. La prueba se realizó para mallas con elementos de tamaño característico iguales a $0.20 \mathrm{~m}$ (no refinadas) y $0.10 \mathrm{~m}$ (refinadas), figura 7. Se observan grandes diferencias entre los residuales para los dos tipos de mallado. Posteriormente, se hizo una comparación con mallas de elementos característicos de $0.15 \mathrm{~m}$ y $0.075 \mathrm{~m}$, hallándose mejores resultados para la malla de $0.10 \mathrm{~m}$.

La figura 8 muestra las diferencias obtenidas empleando los modelos de turbulencia $\kappa-\varepsilon$ y $\kappa-\varepsilon$ low Reynolds en LC. Comparando los tiempos de salida calculados mediante el CFD con los obtenidos en campo (figura 11) se observa que el pico del modelo $\kappa-\varepsilon$ low Reynolds está por encima del medido y se da en un tiempo cercano al obtenido en campo; mientras que en el modelo $\kappa-\varepsilon$ el valor de la concentración máxima del trazador es parecida a la medida en campo y su ocurrencia es posterior a la obtenida en campo. El primer modelo de turbulencia refleja de manera más fiel el tiempo de transporte del trazador; en relación con el valor de la concentración, se debe tener en cuenta que el proceso de centrifugación de las muestras podría haber provocado la pérdida de trazador en la medida.

La siguiente figura ilustra los resultados obtenidos aplicando CFD contrastados con el estudio de dispersión para cada una de las tres topologías.

\section{LBM}

En la figura 9 se observa que en la zona interna los puntos a L/3 del modelo CFD (P11, P12) tienen un comportamiento similar (a y b); igual situación se observa para los puntos a 2L/3 (P21, P22). El trazador viaja más rápido en los estratos intermedios del modelo, a diferencia de lo obtenido en el estudio de campo, en el que el trazador viaja más lento (c y d); sin embargo, se debe anotar que la primera muestra se tomó luego de dos horas de haber inyectado el trazador, por lo que es posible 
Tabla 4. Resumen variables análisis DTR. Ensayo 2

\begin{tabular}{lcccc}
\hline \multicolumn{1}{c}{ Parámetros } & LBM & LC & LB & Unidades \\
\hline $\mathrm{C}_{0}$, concentración inicial & 7.05 & 35.98 & 61.22 & $\mu \mathrm{g}-\mathrm{L}^{-1}$ \\
$\mathrm{t}_{\mathrm{i},}$ tiempo inicial & 4.00 & 2.00 & 2.00 & $\mathrm{~h}$ \\
$\mathrm{C}_{\text {máx }}$ concentración máxima & 37.20 & 35.98 & 61.22 & $\mu \mathrm{g}-\mathrm{L}^{-1}$ \\
$\mathrm{t}_{\mathrm{p}^{\prime}}$ tiempo de máx. concentración & 10.00 & 2.00 & 2.00 & $\mathrm{~h}$ \\
$\mathrm{t}_{0^{\prime}}$ tiempo medio teórico de retención & 114.31 & 131.17 & 131.50 & $\mathrm{~h}$ \\
$\mathrm{t}_{\mathrm{f}}$ tiempo salida total del trazador & 348.00 & 348.00 & 348.00 & $\mathrm{~h}$ \\
$\mathrm{t}_{\mathrm{m}}$, tiempo medio de retención & 59.67 & 110.01 & 50.86 & $\mathrm{~h}$ \\
$\mathrm{t}_{50 \%}$ tiempo salida 50\% del trazador & 52.00 & 72.00 & 40.00 & $\mathrm{~h}$ \\
$\mathrm{t}_{\mathrm{i}} / \mathrm{t}_{0}$ & 0.03 & 0.02 & 0.02 & - \\
$\mathrm{t}_{\mathrm{m}} / \mathrm{t}_{0}$ & 0.52 & 0.84 & 0.39 & - \\
$\mathrm{t}_{\text {térico, }}$ tiempo de retención hidráulica & 100.08 & 95.76 & 95.52 & $\mathrm{~h}$ \\
teórico & & & & \\
Índice corto circuitos & 0.83 & 0.98 & 0.96 & - \\
$\mathrm{e}$ & 2.90 & 2.64 & 2.63 & - \\
Trazador recuperado & 1.93 & 2.42 & 1.52 & $\mathrm{gr}$ \\
\% Trazador recuperado & $50.7 \%$ & $63.8 \%$ & $40.0 \%$ & - \\
\hline
\end{tabular}

Tabla 5. Resumen variables modelo de dispersión y tanques en serie, Ensayo 2

\begin{tabular}{cccc}
\hline Parámetros & LBM & LC & LB \\
\hline$\sigma^{2}$ & 1867.17 & 8457.76 & 1621.42 \\
$\sigma^{2} / \mathrm{t}_{\mathrm{m}}{ }^{2}$ & 0.524 & 0.698 & 0.626 \\
$\delta=\mathrm{D} / \mu \mathrm{l}$ & 0.427 & 0.841 & 0.627 \\
$\mathrm{Pe}$ & 2.34 & 1.18 & 1.59 \\
$\mathrm{n}$ & 1.90 & 1.43 & 1.59 \\
\hline
\end{tabular}

Tabla 6. Condiciones de frontera para el cálculo hidrodinámico

\begin{tabular}{|c|c|c|c|c|c|}
\hline Zona & Tipo de frontera & Valor & Consideraciones LBM & Consideraciones LC & Consideraciones LB \\
\hline Entrada & $\begin{array}{l}\text { Velocidad de } \\
\text { entrada }\end{array}$ & & $\begin{array}{l}0.104\left[\mathrm{~m} \mathrm{~s}^{-1}\right] \\
\text { Intensidad de la } \\
\text { turbulencia, I, } \\
\mathrm{I}=5.43[\%] \\
\text { Diámetro hidráulico = } \\
0.0546[\mathrm{~m}]\end{array}$ & $\begin{array}{l}0.117\left[\mathrm{~m} \mathrm{~s}^{-1}\right] \\
\text { Intensidad de la } \\
\text { turbulencia, I, I = 4.3 [\%]. } \\
\text { Diámetro hidráulico = } \\
0.0508[\mathrm{~m}]\end{array}$ & $\begin{array}{l}\text { Intensidad de la } \\
\text { turbulencia, I, I = } 5.55[\%] \text {. } \\
\text { Diámetro hidráulico = } \\
0.0546[\mathrm{~m}]\end{array}$ \\
\hline Salida & Flujo de salida & 1 & Ídem & $\begin{array}{l}\text { Desbalance permitido en la } \\
\text { masa } 0[\%]\end{array}$ & Ídem \\
\hline $\begin{array}{l}\text { Tubería de } \\
\text { entrada }\end{array}$ & Pared & {$[-]$} & Ídem & “Ley Log” & Ídem \\
\hline $\begin{array}{l}\text { Paredes de } \\
\text { salida }\end{array}$ & Pared & {$[-]$} & Ídem & “Ley Log” & Ídem \\
\hline $\begin{array}{l}\text { Paredes } \\
\text { laterales }\end{array}$ & Pared & {$[-]$} & Ídem & “Ley Log” & Ídem \\
\hline Mallas & Caída en el poro & $\begin{array}{c}0.98\left[\mathrm{~m}^{2}\right] \\
0.01[\mathrm{~m}] \\
0.01\left[\mathrm{~m}^{-1}\right]\end{array}$ & $\begin{array}{l}\text { Permeabilidad } \\
\text { Espesor } \\
\text { Coeficiente de salto de } \\
\text { presión }\end{array}$ & N.A. & N.A. \\
\hline Superficie & Pared & {$[-]$} & Sin esfuerzo cortante & Sin esfuerzo cortante & Ídem \\
\hline
\end{tabular}


Tabla 7. Condiciones para el cálculo del tiempo de inyección y variación de la concentración dentro de los pilotos

\begin{tabular}{|c|c|c|c|c|c|c|}
\hline Zona & $\begin{array}{l}\text { Tipo de } \\
\text { frontera }\end{array}$ & $\begin{array}{l}\text { Valor } \\
{\left[\mathrm{kg} \mathrm{m}^{-3}\right]}\end{array}$ & Unidades & Consideraciones LBM & Consideraciones LC & Consideraciones LB \\
\hline Entrada & $\begin{array}{l}\text { UDS-Valor } \\
\text { específico }\end{array}$ & 0.76 & {$[-]$} & $\begin{array}{l}\text { Tiempo de inyección } \\
20.7 \text { [s]. Después se } \\
\text { hace cero el valor de la } \\
\text { concentración }\end{array}$ & $\begin{array}{l}\text { Tiempo de inyección } \\
24 \text { [s]. Después se hace } \\
\text { cero el valor de la } \\
\text { concentración }\end{array}$ & $\begin{array}{l}\text { Tiempo de inyección } \\
24.55 \text { [s]. Después se } \\
\text { hace cero el valor de la } \\
\text { concentración }\end{array}$ \\
\hline Salida & $\begin{array}{l}\text { UDS-Flux } \\
\text { específico }\end{array}$ & 0 & {$[-]$} & $\begin{array}{l}\text { Desbalance permitido } \\
\text { en la masa } 0[\%]\end{array}$ & & \\
\hline $\begin{array}{l}\text { Tubería de } \\
\text { entrada }\end{array}$ & Sin Flux & {$[-]$} & {$[-]$} & & & \\
\hline $\begin{array}{l}\text { Paredes de } \\
\text { salida }\end{array}$ & Sin Flux & {$[-]$} & {$[-]$} & & & \\
\hline $\begin{array}{l}\text { Paredes } \\
\text { laterales }\end{array}$ & Sin Flux & {$[-]$} & {$[-]$} & & & \\
\hline Superficie & Sin Flux & {$[-]$} & {$[-]$} & & & \\
\hline
\end{tabular}

UDS: Escalar definido por el usuario

que se haya perdido información de una primera concentración máxima. El comportamiento del trazador en la salida es sensiblemente distinto entre CFD ( $a$ y b) y el estudio de dispersión (c y d), no se distingue un pico, aunque la tendencia es similar, la curva respuesta se hace asintótica en un valor cercano a $20 \mathrm{ug}-\mathrm{L}^{-1}$.

Las curvas de concentración visualizadas a la salida del modelo sugieren una mezcla vertical relacionada más con fenómenos difusivos que con fenómenos convectivos. La frontera para la malla, caída en el poro, hizo que el modelo canalizara el flujo a través de los poros y contribuyera a una mejor distribución del trazador, de acuerdo con lo presentado en la figura 10; esta figura indica también que el transporte de RWT en el modelo sigue un comportamiento tipo pistón, pues se observan contornos que muestran una progresión de los valores de concentración en el sentido del flujo, lo cual contrasta con las curvas del estudio de dispersión, las cuales sugieren un flujo con tendencia a RCM. Para un tiempo de 44 horas, luego de la inyección del trazador la canti- dad recuperada a la salida de la LBM en el estudio de dispersión fue de $0.773 \mathrm{mg}$; mientras que en el CFD la cantidad fue de $0.617 \mathrm{mg}$.

\section{LC}

En la figura 11 se observa un comportamiento similar para todos los puntos de la zona intermedia en el modelo CFD (a y b), señalando una mezcla vertical, a diferencia de lo expresado en la curva para el estudio de dispersión (c y d), en el que, además, se presenta un retraso en la aparición de las concentraciones máximas y en la que se observa la ausencia de picos en los puntos superficiales. El comportamiento asintótico se da para los dos casos alrededor de una concentración de 20 ug- $\mathrm{L}^{-1}$. El comportamiento del trazador en la salida es similar tanto para el CFD ( $a$ y b) como para el estudio de dispersión (c y d), aunque se evidencia un corto circuito pronunciado en este último que no sucede en el primero. 
a) Frontera presión de salida

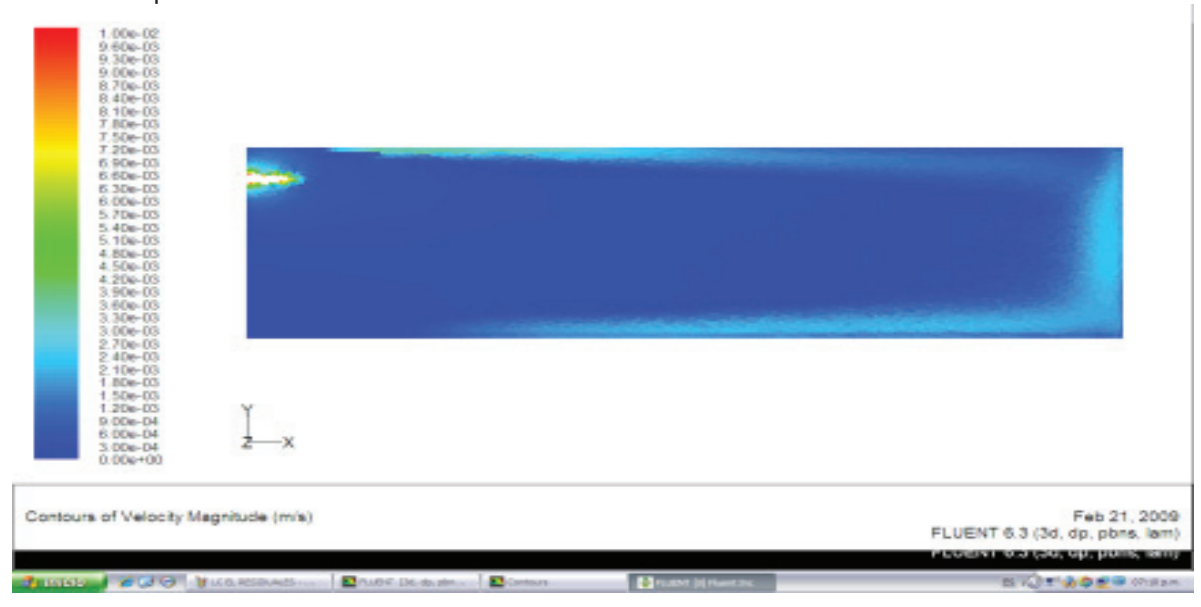

b) Frontera pared

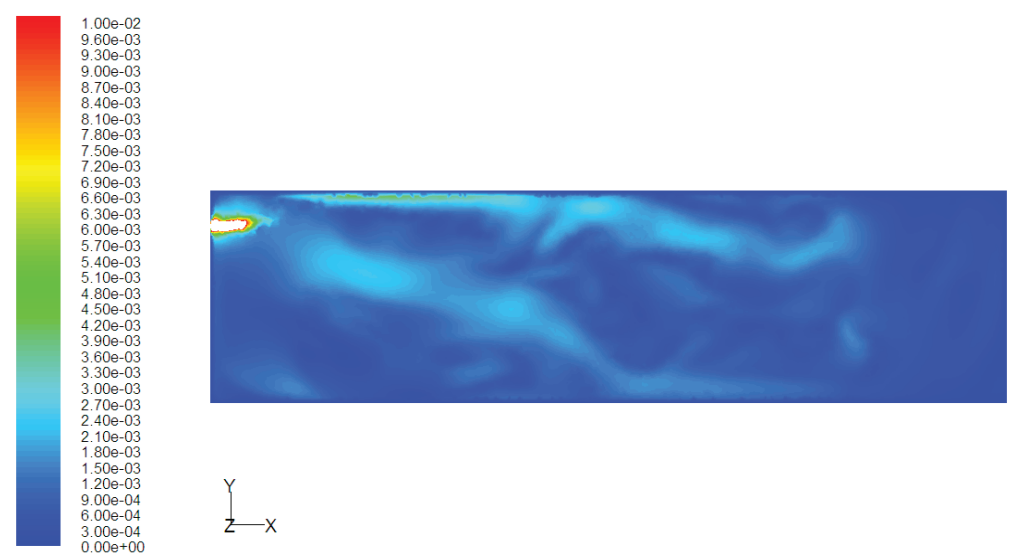

c) Frontera velocidad de entrada
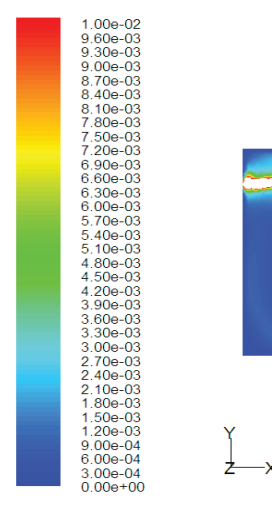

Profiles of Velocity Magnitude $(\mathrm{m} / \mathrm{s})$

Jan 19,2009
FLUENT 6.3 (3d, dp, plons, lam)

Figura 6. Comparación contornos de velocidad distintos tipos de frontera, $\mathrm{m}-\mathrm{s}^{-1}$. LC 
DOI: https://doi.org/10.1016/S1405-7743(14)70661-4

a) Punto de monitoreo. Seguimiento de velocidad en un punto de la laguna

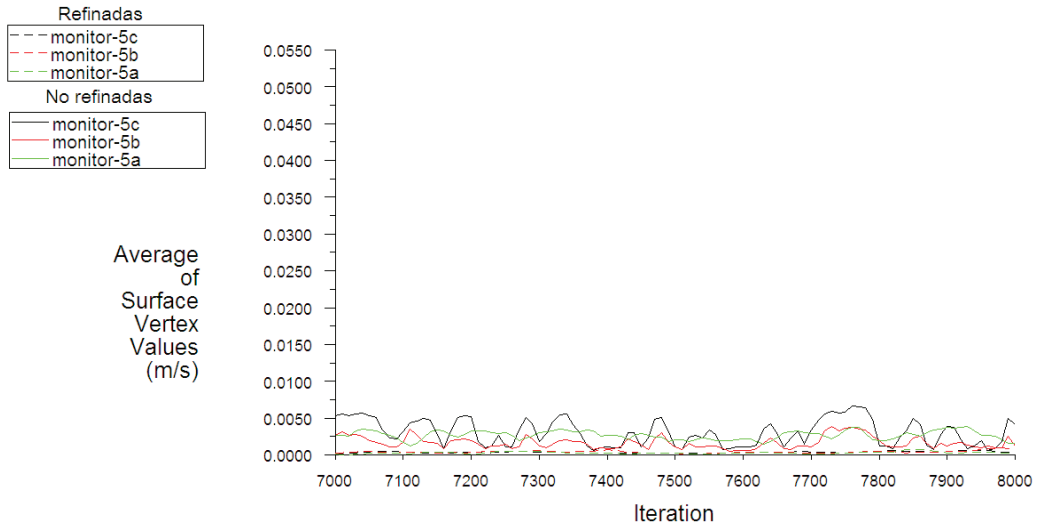

b) Residuales

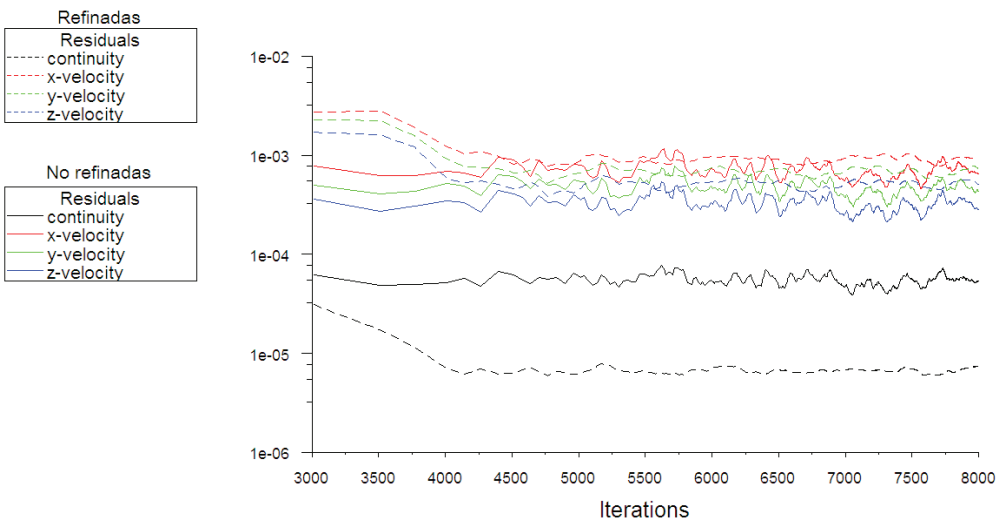

Figura 7. Comparación de puntos de monitoreo y residuales. Frontera pared-Free Slip. LC

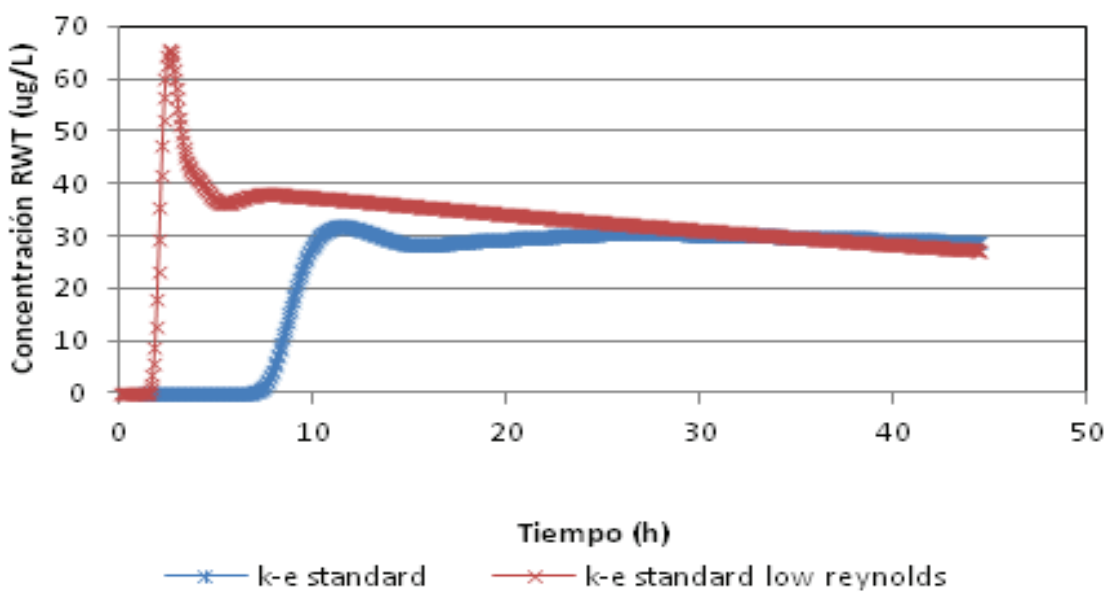

Figura 8. Comparación modelo turbulencia $k-\varepsilon$ y $k-\varepsilon$ low Reynolds. LC 
a) Puntos intermedios

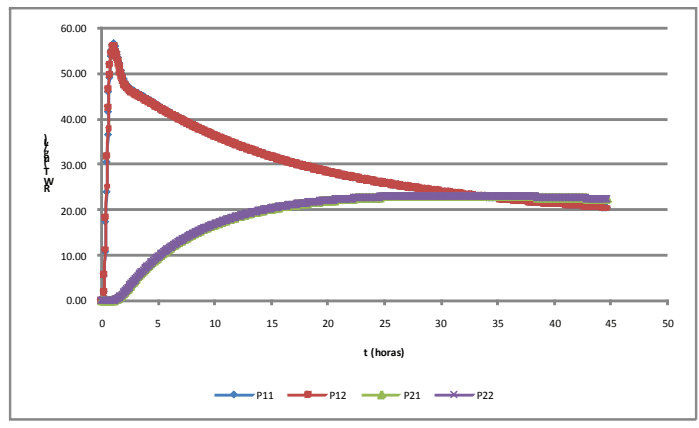

b) Salida

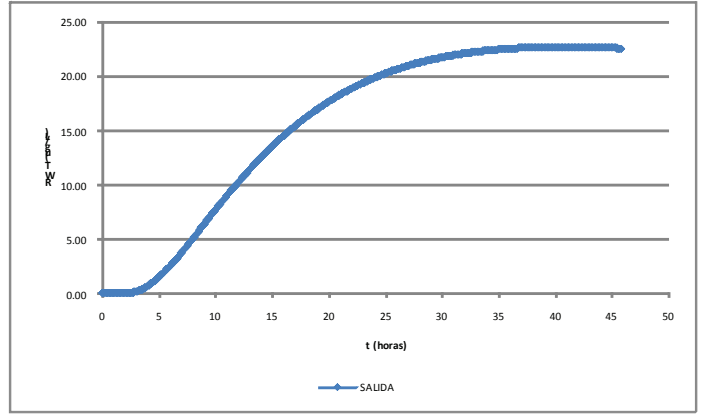

Estudio de dispersión

c) Puntos intermedios

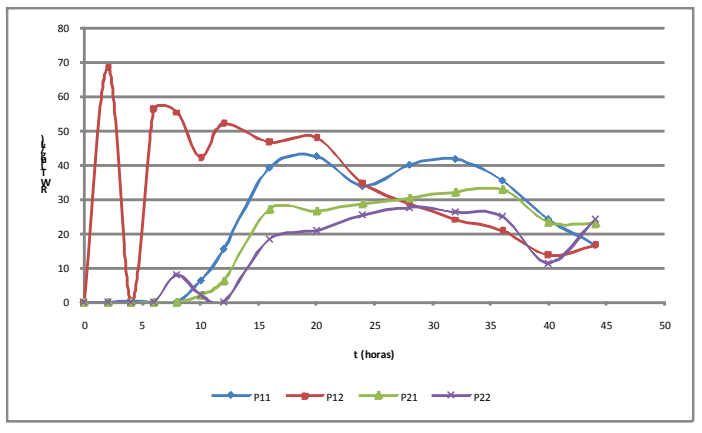

d) Salida

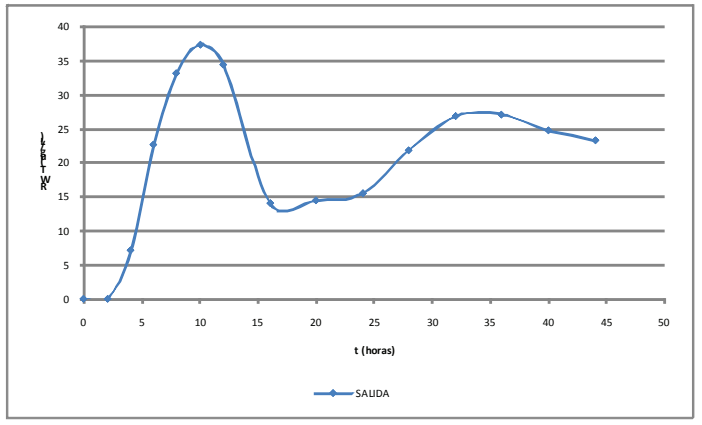

Figura 9. Comparación CFD vs Estudio de dispersión. LBM

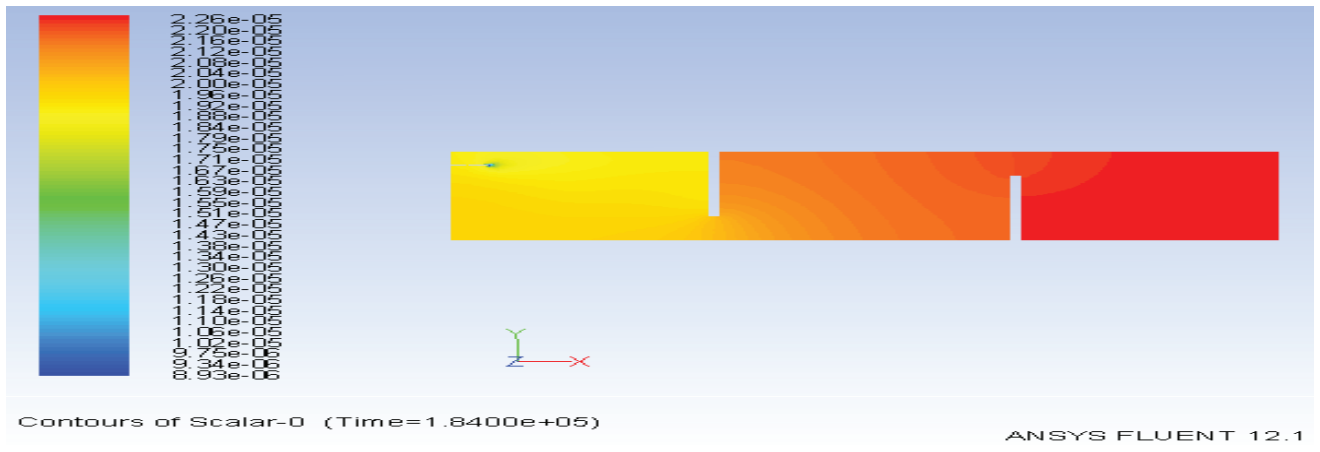

Figura 10. Contornos de RWT a profundidad media. $0.001-22.6$ ug-L ${ }^{-1}$. LBM 
a) Puntos intermedios

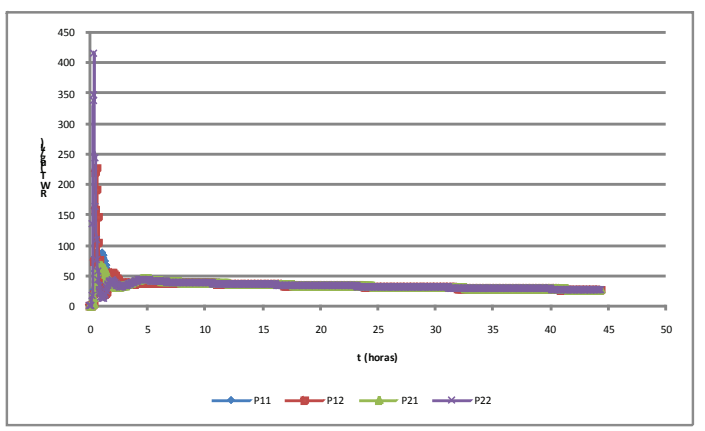

b) Salida

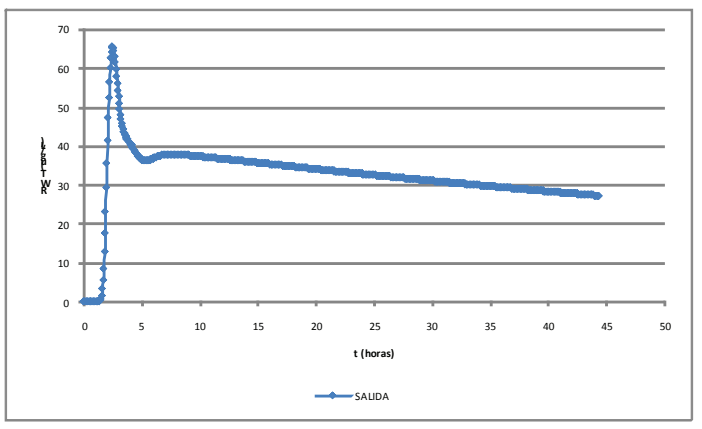

Figura 11. Comparación CFD vs Estudio de dispersión. LC
C) Puntos intermedios

Dispersión

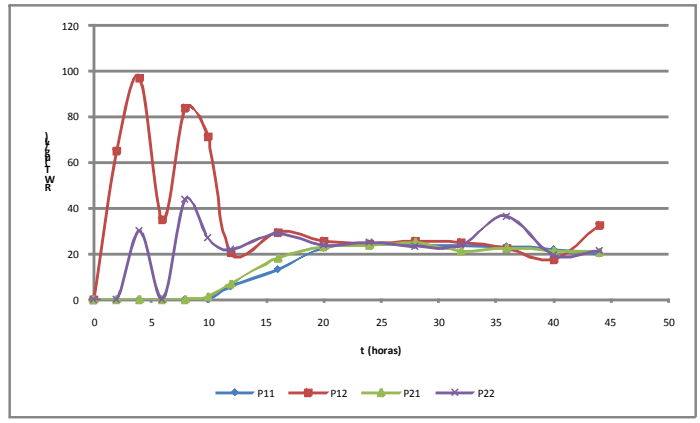

d) Salida

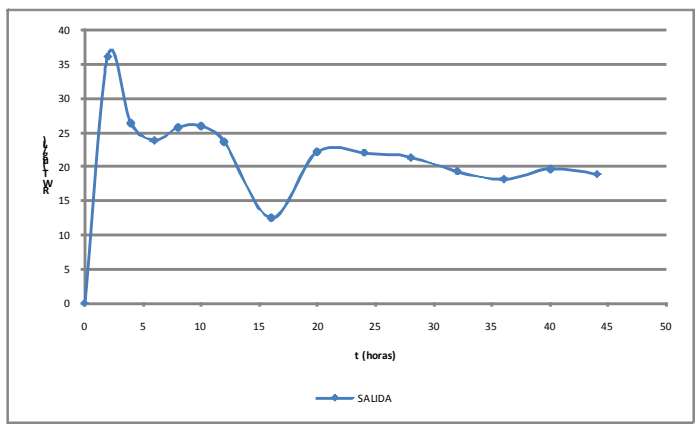

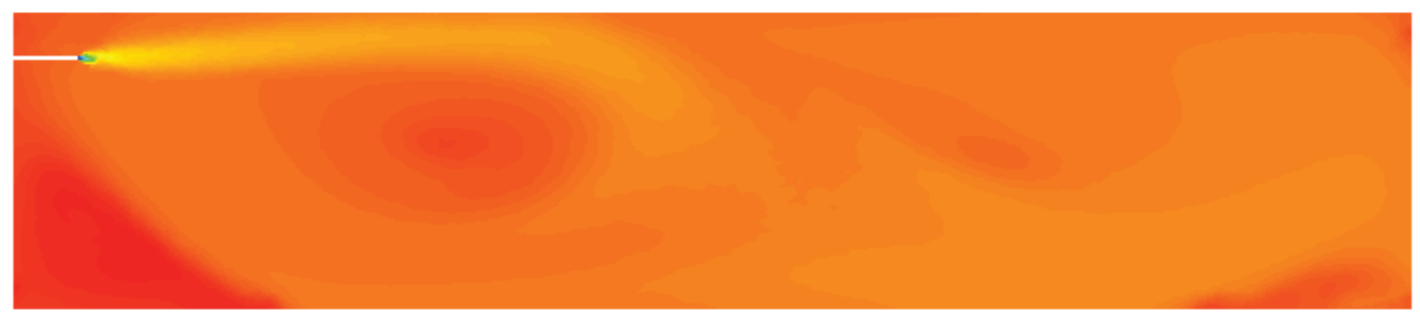

Figura 12. Contornos de RWT a profundidad media. 0.0-31.4 ug/L. LC

La distribución del trazador se ve afectada tanto por la difusión como por la convección, pues no se observa un frente definido de concentración sino distintas áreas o zonas donde se ve retención de la sustancia (figura 12), lo cual es coincidente con lo encontrado en el estudio de dispersión en los puntos internos. Para un tiempo de 44 horas, aproximadamente luego de la inyección del trazador, la cantidad recuperada de RWT a la salida de la LC en el estudio de dispersión y en CFD fueron de 0.745 y $1.04 \mathrm{mg}$, respectivamente.

\section{LB}

El comportamiento del modelo CFD para LB (Figura 13) fue similar al obtenido para LBM, la aparición de las concentraciones máximas en los puntos a profundidad media fue rápida ( $\mathrm{a}$ y $\mathrm{b}$ ), antes de una hora luego de la inyección del trazador, algo que no sucedió en el estudio de dispersión (c y d). Sin embargo, los puntos en superficie sí se comportaron en forma parecida entre el CFD y el estudio de dispersión, tendiendo a valores cercanos a los $20 \mu \mathrm{g}-\mathrm{L}^{-1}$.

En el modelo CFD el pico detectado en el estudio de dispersión no termina de generarse y los fenómenos difusivos se imponen a los convectivos, pues la RWT no se transporta de manera similar a lo hallado en el estudio de campo y tarda mucho en salir. El efecto de los bafles es retrasar la salida del trazador y se evidencia en el estudio de dispersión, pero en el modelo CFD se sucede de manera exagerada, generándose una zona de 
CFD

a) Puntos intermedios

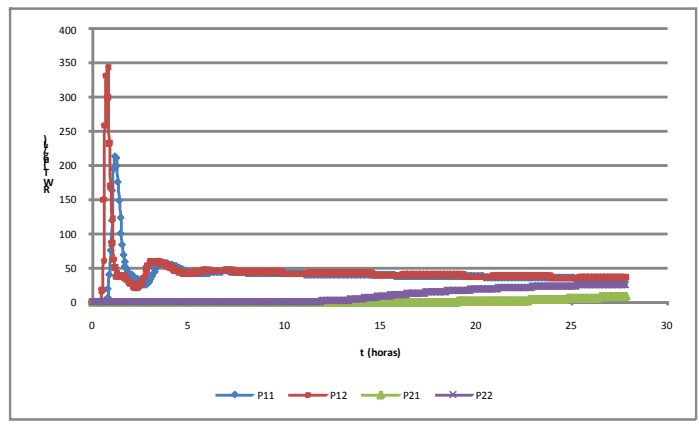

b) Salida

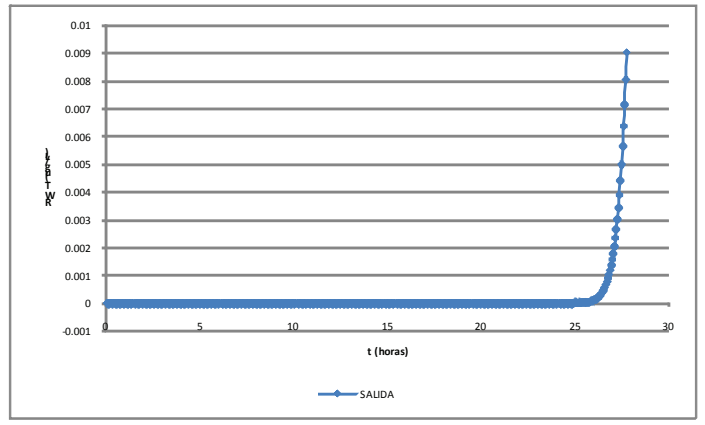

Figura 13. Comparación CFD vs Estudio de dispersión. LB

alta concentración en la zona intermedia del segundo compartimiento (figura 14); esta figura muestra un grado de mezcla menor al obtenido en LC y en LBM. Para un tiempo de 28 horas, aproximadamente luego de la inyección del trazador, las cantidades recuperadas de RWT a la salida de la LB en el estudio de dispersión y en CFD fueron de 0.984 y $0.003 \mathrm{mg}$, respectivamente.

En los casos, LBM y LC, el trazador tiende a valores similares tanto a nivel de CFD como de estudios de dispersión, cercanos a $20 \mathrm{ug}-\mathrm{L}^{-1}$. El comportamiento de los pilotos en los estudios de dispersión indicó la presencia de cortos circuitos atribuibles a factores como dirección y magnitud del viento, presencia de biomasa (Shilton $e t$ al., 2008), así como cambios en la densidad de la temperatura del agua. Es de resaltar que este fenómeno también se observó en los puntos intermedios, no solo en la salida; en las capas intermedias del piloto se aprecia mejor el fenómeno de corto circuitos, mientras en el modelo CFD no, apareciendo solo un pico y no varios. Las diferencias en las curvas del trazador en los puntos de la zona intermedia de los modelos CFD entre los tres pilotos señalan que los dispositivos bafles y la malla modifican el comportamiento del fluido, especialmente
Dispersión

c) Puntos intermedios

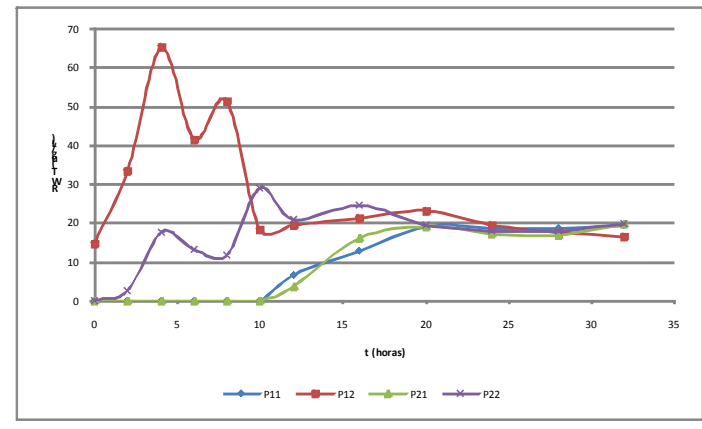

d) Salida

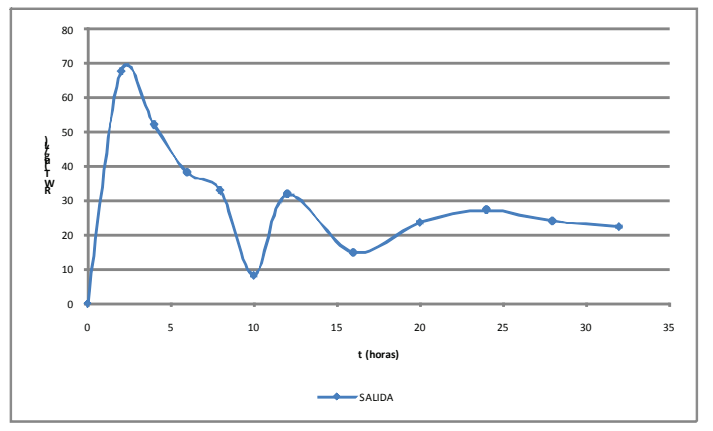

para los puntos localizados a profundidad media. De igual manera sucede en la salida, el comportamiento del trazador es distinto, reforzando la idea de que las modificaciones afectan el transporte del fluido. Los puntos de la zona intermedia en los tres pilotos modelados muestran diferencias entre LBM-LB y LC para los puntos superficiales, mientras que el comportamiento para los puntos a profundidad media es similar en las tres LFS. Según el estudio de dispersión, el efecto de bafles y mallas en LBM y LB afectó el transporte de la RWT, especialmente a profundidad media en las que se ve un retraso en la aparición de las concentraciones máximas, como si se presentaran cortos circuitos en un plano a esa profundidad. Es importante señalar aquí que los caudales variaron durante el estudio de dispersión y esto pudo afectar el transporte del trazador en las unidades, más si se tiene en cuenta que presentan retroflujos y zonas muertas (Shilton et al., 2008; Shilton y Harrison, 2003) como las evidenciadas en las curvas DTR; además, los cortos circuitos podrían explicarse por las diferencias de densidad del agua a distintas profundidades, ya que las diferencias de temperaturas que se detectaron durante el estudio de trazadores en las tres 


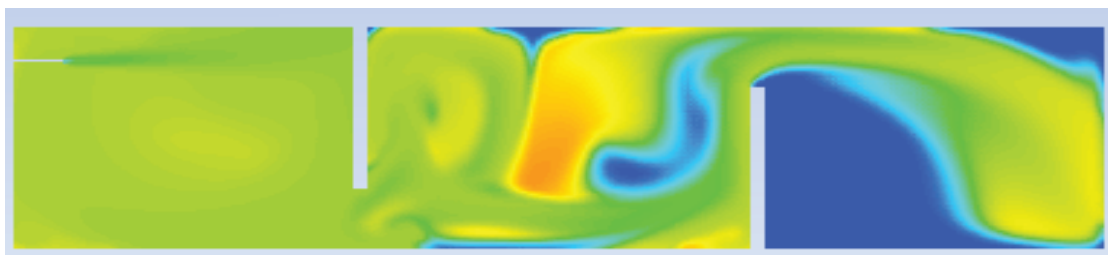

Figura 14. Contornos de RWT a profundidad media. 0.0-40.0 ug- $\mathrm{L}^{-1}$. LB unidades piloto, variaron entre $\pm 4^{\circ} \mathrm{C}$ dependiendo de la hora del día. Se hizo un chequeo de la condición de viento sobre la superficie de la laguna en el modelo CFD para LC (figura 15).

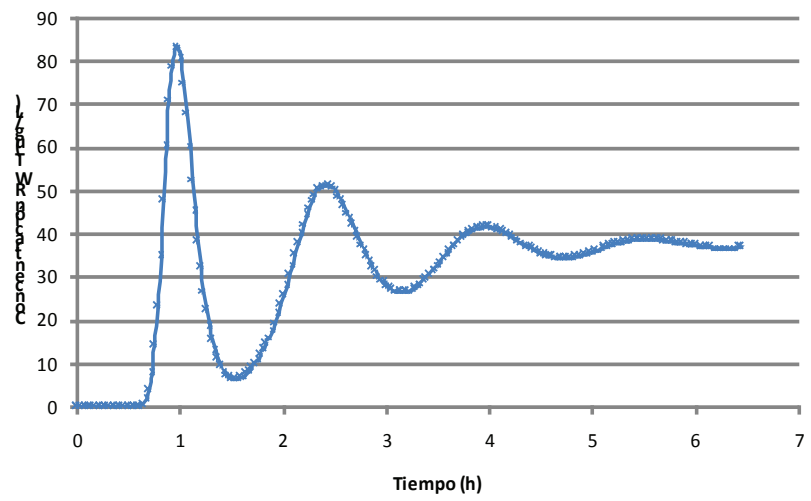

Figura 15. Modelado para condición de viento. LC

El resultado indicó que el valor de concentración máxima superó el obtenido para la condición sin viento y por consiguiente lo medido en campo. El efecto del viento sobre el fluido es significativo, en el sentido de que cambia la magnitud y la forma de salida del trazador, generando un efecto parecido al detectado en el estudio de dispersión llevado a cabo en este piloto. La rosa de los vientos para las horas en las que se presenta la información de CFD y el estudio de dispersión se presenta en la figura 16, así como la orientación de las unidades piloto y el sentido del flujo (flecha verde).

La dirección predominante del viento fue en el sentido del flujo, acelerando el transporte del fluido en las LFS; el efecto del viento se muestra como posible causante de cortos circuitos en los pilotos por su efecto sobre las capas superficiales de fluido, algo que quedó reflejado en los estudios de dispersión aunque en menor grado que en el CFD de la LC. Lo anterior, hace necesario que los estudios de CFD y los de modelado en general de un estudio de dispersión no sean subvalorados (Alvarado et al., 2012), para que se consideren como un elemento que afecta la representación que se hace del fluido.

Como se observa, el modelo CFD no mostró diferencias significativas para los puntos P11, P12 y P21 en LBM; igual sucedió para los puntos 1.2 en LC y LB. Estas diferencias podrían atribuirse al hecho de que el modelado computacional no incluyó efectos como el ingreso no instantáneo de la RWT, la cual ingresó en forma gradual al piloto, tardando posiblemente un poco más de tiempo que el calculado para la inyección del trazador en el modelo CFD, en este sentido otros autores (Shilton et al., 2008) han expresado algo similar. a)

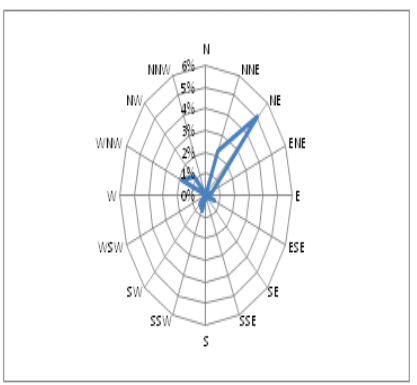

b)

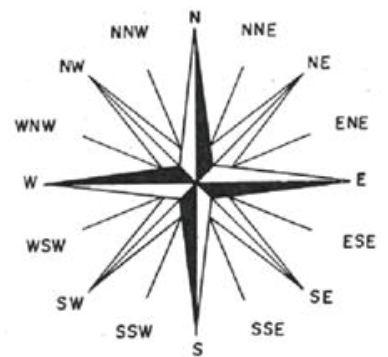

C)

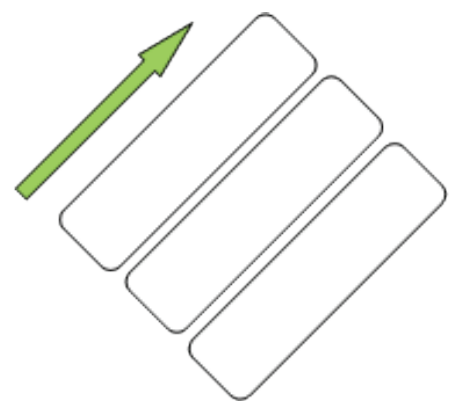

Figura 16. Rosa de los vientos y sentido de flujo en las unidades piloto 
Tabla 8. Prueba t-pareada CFD-Estudio de Dispersión

\begin{tabular}{lclclc}
\hline \multicolumn{1}{c}{ LBM } & $\begin{array}{c}\text { Sig. } \\
(2 \text {-colas })\end{array}$ & \multicolumn{1}{c}{ LC } & $\begin{array}{c}\text { Sig. } \\
(2 \text {-colas })\end{array}$ & LB & Sig. \\
\hline P11CFD - P11EDISP & .147 & LCP11CFD - LCP11EDISP & .000 & LBP11CFD - LBP11EDISP & .000 \\
P12CFD - P12EDISP & .307 & LCP12CFD - LCP12EDISP & .350 & LBP12CFD - LBP12EDISP & .290 \\
P21CFD - P21EDISP & .612 & LCP21CFD - LCP21EDISP & .000 & LBP21CFD - LBP21EDISP & .062 \\
P22CFD - P22EDISP & .042 & LCP22CFD - LCP22EDISP & .010 & LBP22CFD - LBP22EDISP & .010 \\
SALIDACFD - & .027 & LCSALIDACFD - & .000 & LBSALIDACFD - & .001 \\
SALIDAEDISP & & LCSALIDAEDISP & & LBSALIDAEDISP & \\
\hline
\end{tabular}

$\alpha$ igual a 0.05

\section{Conclusiones}

El efecto de un dispositivo como la malla promueve no solamente el retraso en la salida del trazador por efecto de fijación de biomasa, sino que en los límites de la frontera podría generar condiciones de turbulencia distintas en el fluido; por lo que en el caso de un modelado CFD es necesario ajustar los parámetros de la frontera en el modelo para que su representación de la realidad del piloto sea más acertada. Por otro lado, los cambios de temperatura y la condición de viento sobre la superficie de una laguna se pueden estudiar a profundidad realizando el modelado con esas condiciones, de allí que se sugiera no subestimarlo en estudios similares.

\section{Agradecimientos}

El autor agradece al Departamento Administrativo de Ciencia, Tecnología e Innovación, Colciencias, Colombia, a ACUAVALLE S.A. ESP y a la Universidad del Valle por el apoyo financiero y logístico de la investigación.

\section{Referencias}

Alvarado A., Vedantam S., Goethals P., Nopens I. A Compartmental Model to Describe Hydraulics in a Full-Scale Waste Stabilization Pond, Water Research, volumen 46 (número 2), 2012: 521-530.

ANSYS. FLUENT 6.3 User's Guide: Fluent Inc., 2006. Banda C. Modern Design of Waste Stabilization Ponds in Warm Climates: Comparison with Traditional Design Methods, University of Leeds, Leeds, 2003.

Banda C. Modern Computational Fluid Dynamics Modelling Baffled Waste Stabilization Ponds, University of Leeds, United Kingdom, 2007.

Bird R.B., Stewart W.E., Lightfoot E.N. Fenómenos de transporte, 2a ed., México, Limusa Wiley, 2006.

Branncock M. Computational Fluid Dynamics Tools for the Design of Mixed Anoxic Wastewater Treatment Vessels, (unpublished thesis), Universidad de Queensland, 2003.
Crites R., Tchobanoglous G. Sistemas de manejo de aguas residuales para núcleos pequeños y descentralizados, vol I, Colombia, McGraw Hill, 2000.

DAVIS. Wireless Vantage Pro2 ${ }^{\mathrm{TM}} \&$ Vantage Pro2 ${ }^{\mathrm{TM}}$ Plus Stations. Console Manual, Editado por Hayward, CA., DAVIS Inc., 2008.

Dimotakis P.E. Turbulent Mixing, Annu. Rev. Fluid. Mech., volumen 37, 2005: 329-356.

Ferziger J.H., Peric M. Computational Methods for Fluid Dynamics, Alemania, Springer, 2002.

Han X., Sagaut P., Lucor D. On Sensitivity of RANS Simulations to Uncertain Turbulent Inflow Conditions, Computers \& Fluids, volumen 61, 2012: 2-5.

Jakeman J.A., Letcher R.A., Norton J.P. Ten Iterative Steps in Development and Evaluation of Environmental Models. Environmental Modelling \& Software, volumen 21, 2006: 602-614.

Ji Z.G. Hydrodynamics and Water Quality. Modeling Rivers, Lakes, and Estuaries, Wiley-Interscience, Nueva Jersey, John Wiley \& Sons, 2008.

Kilpatrick F. Dosage Requirements for Slug Injections of Rhodamine BA and WT Dyes, en: Geological Survey Research, Estados Unidos, 1970.

Kilpatrick F., Wilson J.J. Mesurement of Time of Travel in Streams by Dye Tracing, revision of the manual Mesurement of Time of Travel in Streams by Dye Tracing, en: Book 3, (Geological Survey Techniques of Water Resources Investigations). Estados Unidos, 1989, pp. 27.

Laín S. Modelado y simulación de flujos inducidos por burbuja, Colombia, Universidad Autónoma de Occidente, 2007.

Levenspiel O. Chemical Reaction Engineering, John Wiley \& Sons, 1999.

Mara D.D. Domestic Wastewater Treatment in Developing Countries, Earthscan/James \& James, UK, 2004.

Patankar S. Numerical Heat Transfer and Fluid Flow. Estados Unidos, Taylor \& Francis, 1980.

Sah L. 3D Modelling of Secondary Facultative Ponds, (unpublished thesis), UNESCO-IHE, Delft, 2009.

Shilton A. Studies into the Hydraulics of Waste Stabilisation Ponds, (unpublished Ph.D thesis), Massey University, Nueva Zelanda, 2001. 
Shilton A., Harrison J. Guidelines for the Hydraulic Design of Waste Stabilization Ponds, Institute of Technology and Engineering, Palmerston North, Nueva Zelanda, 2003.

Shilton A., Kreegher S., Grigg N. Comparison of Computation Fluid Dynamics Simulation against Tracer Data from a Scale Model and Full-Sized Waste Stabilization Pond, Journal of Environmental Engineering, volumen 134, 2008: 845-850.

Treybal R.E. Operaciones de transferencia de masa, 2a ed., Mc GrawHill, México, 1980.

Von-Sperling M. Design of Facultative Ponds Based on Uncertainly Analysis, Water Science Technology, volumen 33, 1996: 41-47.

Yánez F. Lagunas de estabilización. Teoría, diseño y mantenimiento, Imprenta Monsalve, Cuenca, Ecuador, 1993.

\section{Este artículo se cita:}

\section{Citación estilo Chicago}

Aponte-Reyes, Alexander. Validación de modelos hidrodinámicos de tres modelos topológicos de lagunas facultativas secundarias. Ingeniería Investigación y Tecnología, XV, 04 (2014): 637-654.

\section{Citación estilo ISO 690}

Aponte-Reyes A. Validación de modelos hidrodinámicos de tres modelos topológicos de lagunas facultativas secundarias. Ingeniería Investigación y Tecnología, volumen XV (número 4), octubrediciembre 2014: 637-654.

\section{Semblanza del autor}

Alexander Aponte-Reyes. Es ingeniero sanitario con maestría en ingeniería sanitaria y ambiental, actualmente es candidato a doctor en la misma área. Sus áreas de trabajo son el abastecimiento de agua y el control de la contaminación ambiental a través de un enfoque ecológico, incluyendo proyectos de investigación y desarrollo sobre tecnologías naturales, el empoderamiento de la comunidad y el comportamiento higiénico. Su perfil profesional incluye proyectos de investigación y la experiencia en las actividades relacionadas con la promoción y ejecución de proyectos de desarrollo, orientados hacia las zonas rurales, municipios pequeños y medianos. 